\title{
An Isolator System for minimally invasive surgery: the new design
}

\author{
Tim Horeman • Frank-Willem Jansen • \\ Jenny Dankelman
}

Received: 25 August 2009/Accepted: 19 December 2009/Published online: 28 January 2010

(C) The Author(s) 2010. This article is published with open access at Springerlink.com

\begin{abstract}
Background The risk of obtaining a postsurgical infection depends highly on the air quality surrounding the exposed tissue, surgical instruments, and materials. Many isolators for open surgery have been invented to create a contained sterile volume around the exposed tissue. With the use of an isolator, a surgical procedure can be performed outside sterile environments. The goal of this study was to design an Isolator System (IS) for standard laparoscopic instruments while instrument movements are not restricted. Methods The developed IS consists of a sleeve to protect the instrument shaft and tip and a special balloon to protect the incision and trocar tube. A coupling mechanism connected at the sleeve allows instrument changes without contamination of the isolated parts. Smoke tests were performed to show that outside air does not enter the new IS during a simulated laparoscopic procedure. Eight test runs and one baseline run inside a contained volume filled with thick smoke were performed to investigate whether smoke particles entered the Isolator System. Filters were used to identify smoke entering the Isolator System.

Results Seven filters showed no trace of smoke particles. In one test run, a part of the IS loosened and a small brown spot was visible. The filter from the baseline run was completely covered with a thick layer of particles, proving
\end{abstract}

\section{T. Horeman $(\varangle) \cdot$ J. Dankelman}

Department of BioMechanical Engineering, Faculty

of Mechanical, Maritime and Materials Engineering ( $3 \mathrm{mE})$,

Delft University of Technology, Mekelweg 2, 2628 CD Delft,

The Netherlands

e-mail: T.Horeman@tudelft.nl

F.-W. Jansen

Department of Gynecology, Leiden University Medical Center, Leiden, The Netherlands the effectiveness of the test. During all test runs, the isolated instrument was successfully locked on and unlocked from the isolated trocar. Instrument movements gave no complications. After removal of the isolated instrument, it took three novices an average of 3.1 (standard deviation (SD), 0.7) seconds to replace it correctly on the isolated trocar.

Conclusions The designed IS for laparoscopy can increase sterility in environments where sterility cannot be guaranteed. The current design is developed for laparoscopy, but it can easily be adapted for other fields in minimally invasive surgery.

Keywords Laparoscopy $\cdot$ Minimally invasive surgery · Isolator systems · Infection prevention · Trocar friction

\section{Postsurgical infections}

Postsurgical infections (PSI) are still common after minimally invasive surgery. A review of 16 articles on the subject revealed an average of $4.02 \%$ intra-abdominal infections after laparoscopic surgery [1]. A PSI does not only cause the patient great discomfort but could become life threatening if the patient is in poor condition [2-10]. Infections can occur if the patient's exposed tissue makes contact with airborne particles or surfaces carrying harmful microorganisms. One way to reduce the risk of infection is to minimize contact between incision, instruments, and possibly contaminating air or surfaces [1]. In well-resourced hospitals, this goal is realized by strictly controlling the environment in the operating room. Expensive air control devices are installed to create laminar air flow and the operating rooms are pressurized. Furthermore, special clothing and disposable covering material for medical 
equipment are used to increase safety. To reduce the risk of infection even further, antibiotics are used before or after surgery. In some hospitals in underdeveloped countries, antibiotics are used in more than $80 \%$ of all surgical procedures [11]. Despite these and other precautions, infections still cause a major financial burden that may be as much as $\$ 4,000$ per case [2-5].

\section{Surgical isolators}

Instead of reducing the number of potentially dangerous particles, microorganisms, or viruses inside the entire operating room, it is possible to create a small, sterile environment around the patient or surgical area (Fig. 1). This small, sterile environment can be useful if surgery is performed inside a nonsterile environment or if a patient is infected with a contagious disease and contamination of the environment needs to be prevented. Already at the beginning of the 20th century, surgeons, bacteriologists, and technicians experimented with partially isolated sterile environments in which surgical procedures were performed. The design of these "isolators" depended on diverse aspects, such as body (part) length, the procedure performed, and the environment [12-22]. Most of the experimental isolators effectively decreased the risk on PSI or contamination of the environment but were never successfully commercialized. Some surgeons suggest that current isolators often are not used because they are not user-friendly, are time consuming, and they restrict vision and arm movement during surgery [23, 24].

\section{Minimally invasive surgery isolator}

Usually, instruments for minimally invasive surgery (MIS) are guided by trocars. They allow endoscopic instruments to move with 4 degrees of freedom (DOF) and without

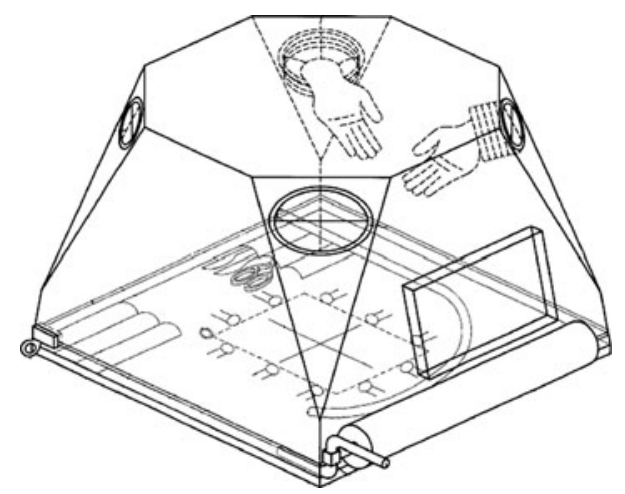

Fig. 1 Schematic of an Isolator System for open surgery on the abdominal cavity [18]

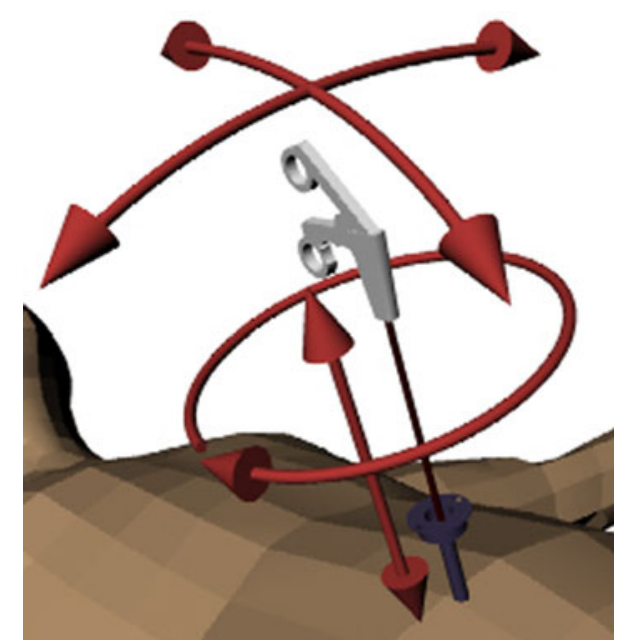

Fig. 2 Movement in 4 DOF of an instrument guided by a trocar

friction between instrument and anatomic layers (Fig. 2). Most trocars are equipped with valves that prevent outflow of gas from the pneumoperitoneum. For each individual MIS procedure, trocars are strategically placed to allow endoscopic instruments to reach and manipulate tissue where needed inside the anatomic cavity. If instrument, trocar, anatomic cavity, and incision are isolated from the environment at all time, the risk of contamination of the patient or contamination of the environment caused by a contagious-diseased patient could drastically decrease. However, large movements of long instruments around the incisions make existing isolators difficult or, in most cases, impossible to use.

An isolator, specially designed for standard endoscopic instruments, could increase safety while movements of the instruments are not restricted. During use, the procedure performed with the isolator must resemble the same procedure performed during common practice. Furthermore, the risk of complications other than infection should not increase. Because the chance of surgical errors seems related to the mental load of the surgeon and staff, it is obvious that a new isolator should be intuitive to use and not add extra complexity to the procedure or operating room $[25,26]$. This new type of isolator might be the solution for MIS performed in underdeveloped areas, outpatient clinics, or intervention rooms where poor air conditions can cause infections. In case of contagious diseases, such as hepatitis or HIV, the Isolator System could protect the operating room and personnel against contaminated tissue and migrating cells coming from the patient.

\section{Materials and methods}

The current research consists of two parts. The first goal is to design a simple and low-cost new Isolator System for 


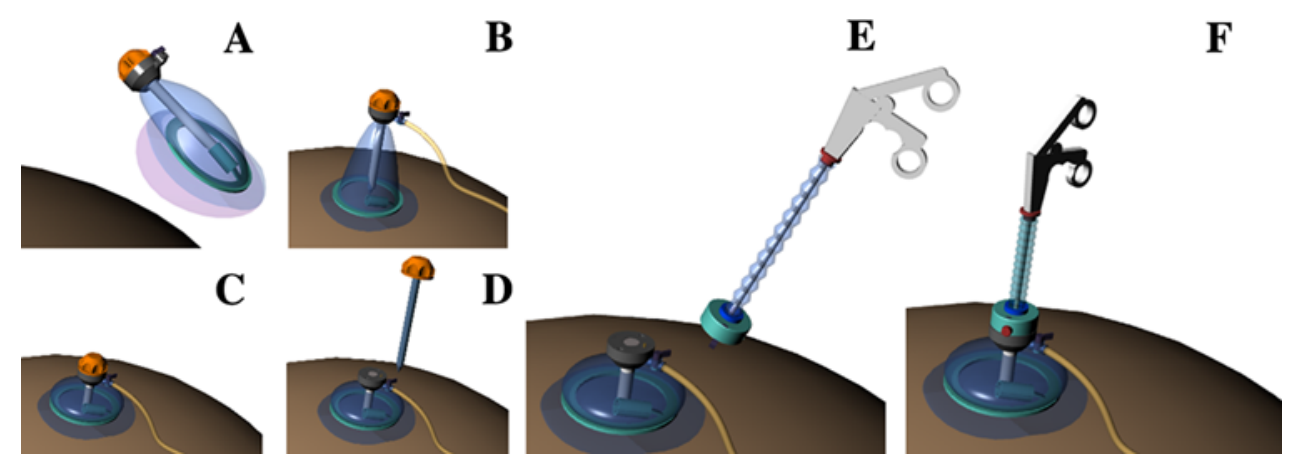

Fig. 3 Placement of the sterile trocar in chronological order. A After the incision is made inside the abdomen, the protection layer is peeled from the Trocar Isolator pad. B Pad is placed over the incision. C
Trocar is pressed trough the Trocar Isolator pad and manipulated trough the different tissue layers. D Trocar pin is removed. $\mathbf{E}$ and $\mathbf{F}$ Isolated instrument is locked on the isolated trocar
MIS. The second goal is to test the functionality of this Isolator System, especially built for laparoscopic procedures.

\section{Isolator System development}

For qualification of the functionality of the new Isolator System for laparoscopy, a number of design specifications were established. The most important design specifications are listed in the first and second column of Table 1.
In the literature, knowledge about design specifications required for isolators for MIS is not available. During a literature study, different instruments, trocar systems and methods for common minimally invasive procedures were investigated. Forces, torques, and movements were measured during a simulated laparoscopic procedure performed with the commonly used Endopath Excel ${ }^{\circledR}$ trocar system in combination with a standard laparoscopic instrument. To see if the new Isolator System does not increase complexity during surgery, a laparoscopic procedure performed with

Table 1 Most important design specifications

\begin{tabular}{|c|c|c|}
\hline Design requirements & Required value & Actual value \\
\hline Inserting instrument ${ }^{\mathrm{a}}$ & 1 hand & $1-2$ hands \\
\hline Removal instrument & 2 hands & 2 hands \\
\hline $\operatorname{Mass}(\mathrm{g})^{\mathrm{b}}$ & Max. 500 & 270 \\
\hline Movements, instrument in trocar & $4 \mathrm{DOF}$ & $4 \mathrm{DOF}$ \\
\hline Rotations around incision & $140^{\circ}$, all directions & $>140^{\circ}$, all directions \\
\hline Axial movements, instruments & $80 \%$ of shaft length & $90 \%$ of shaft length \\
\hline Axial rotations around instrument shaft & $360^{\circ}$ & $>360^{\circ}$ \\
\hline Interface & 1 manual actuator (button/handle/etc.) & 1 button \\
\hline Time to install or remove instrument & $<5 \mathrm{~s}$ & $\begin{array}{l}<4 \text { s install } \\
<1 \text { s remove }\end{array}$ \\
\hline Extra preparation time ${ }^{\mathrm{c}}$ & $<10 \min$ & $<3 \min$ \\
\hline Particle reduction $^{\mathrm{d}}$ & $>95 \%$ & $\approx 100 \%$ \\
\hline Friction instrument during axial movements & $0.35 \mathrm{~N}$ & $<0.05 \mathrm{~N}$ \\
\hline Applied pressure on manual actuator $\left(\mathrm{N} / \mathrm{mm}^{2}\right)$ & 1 & $<0.5$ \\
\hline Instrument changes (times per system) & 20 & $>20$ \\
\hline Shaft length instruments (mm) & Max. 350 & Max. 350 \\
\hline Shaft diameter instruments $(\mathrm{mm})$ & Max. 11 & Max. 11 \\
\hline
\end{tabular}

DOF degrees of freedom

a Two hands are required when the isolated instrument is placed without visual feedback

b Mass includes the complete Isolator System, including trocar pin and tube for three instruments

${ }^{c}$ Time spend inside the operating room (OR); extra time required to wrap and sterilize the instruments outside the OR is not included

${ }^{\mathrm{d}}$ Percentage based on difference between particles found on baseline-run filter (without isolator) and test-run filters (with isolator)

${ }^{\mathrm{e}}$ No particles found on the filters of the seven successful test runs (after the second unsuccessful run, the design has been improved) 
the new Isolator System must be comparable with a procedure performed without the Isolator System. The number of instrument changes is estimated at 20 per procedure per trocar. To keep the Isolator System portable and lightweight it should not weigh more than $500 \mathrm{~g}$ in total. If a simple manual actuator, e.g., button or handle, is used to install or remove the isolator, operating this actuator should not require more than one hand. The best concept developed during the design process is presented in Fig. 3.

The complete system is built around a newly developed trocar system and consists of two parts: Part 1, called "Shaft Isolator," contains the upper flange, isolator foil, and coupling; Part 2, called "Trocar Isolator," consists of a trocar protected by a balloon-like isolator filled with gas. Figure $3 \mathrm{~A}-\mathrm{F}$ presents how trocar placement can be performed without contact between trocar pin, trocar tube, and potentially contaminating air. When the trocar is not inserted, the trocar shaft, which penetrates the tissue layers, is surrounded by sterile gas. Before the procedure starts, the skin is disinfected and the incision is made. The protection layer is peeled off the sticky pad (Fig. 3A) and the Trocar Isolator is pressed onto the clean skin (Fig. 3B). The trocar with trocar tube is now inserted through the tip holder and pad of the Trocar Isolator and tissue layers (Fig. 3C). The trocar is ready for use after the trocar pin has been removed (Fig. 3D).

The coupling should ensure that outside air never contaminates the isolated volume. Therefore, a safety pin is introduced that locks the instrument inside the Shaft Isolator when the coupling is not correctly placed on the trocar (Fig. 3E). If the coupling is correctly placed on the isolated trocar, it locks automatically and allows the instrument to enter the isolated trocar and anatomic cavity (Fig. 3F). By pressing the single button on the coupling, the isolated instrument can be removed from the isolated trocar if the instrument is entirely withdrawn into the Shaft Isolator. If the instrument's shaft or tip is not entirely withdrawn into the Shaft Isolator, the safety pin of the coupling is obstructed and the button cannot be pressed. The location of the button on the coupling makes it possible to unlock the system with only one hand. All parts of the prototyped coupling and trocar were manufactured with machinery from the Delft University of Technology. The thin isolating thermoplastics were shaped by hand and the seal of the Shaft Isolator valve was made from a rubber "cofferdam" used in dentistry. All of the materials used were low cost and easy to acquire.

\section{Isolator System evaluation}

The goal was to design an Isolator System for laparoscopic surgery that does not restrict the surgeon during the laparoscopic procedure. However, extra effort is needed to lock

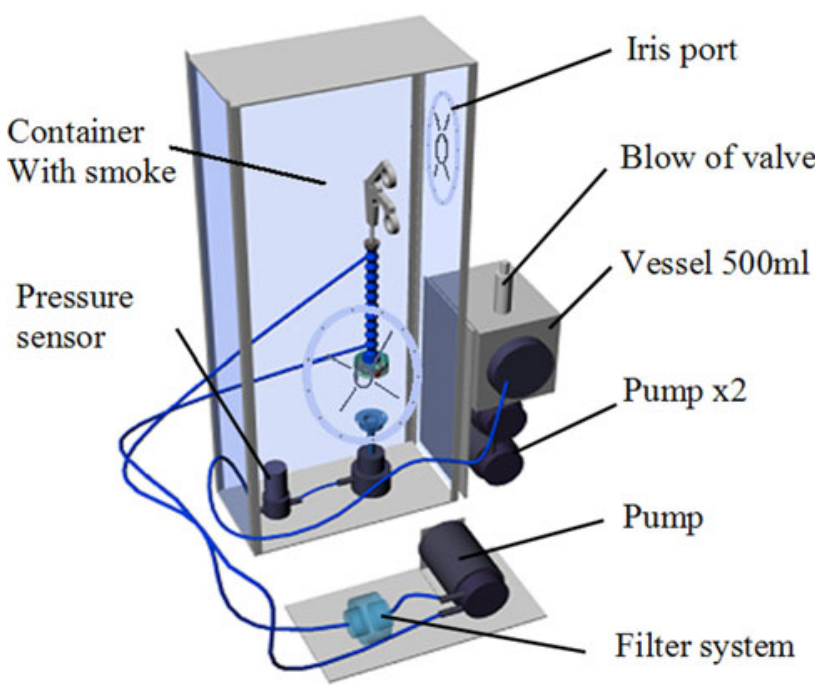

Fig. 4 Smoke test setup: hardware overview

and unlock the system. Therefore, it is important to check the dexterity and functionality of the design.

Test setup—smoke test

The smoke test setup consists of a smoke-filled outer volume surrounding a separate internal volume that circulates over a filter. This internal circulated volume contains an isolated standard laparoscopic instrument that can be locked on a pressurized trocar during testing. A container was built from 1-mm-thick transparent Lexan to contain the smoke around the tested Isolator System. In this container, two iris ports provided access to the Isolator System. Air was used to pressurize the trocar. The pressure was generated by two diaphragm pumps (Rietschle Thomas Netherlands BV, Woerden, Netherlands). During the smoke tests, the pressure on the system was adjustable with a faucet and a custom made blow-off valve. The pressure was measured directly at the foot of the trocar with an ATM pressure sensor (AEsensors BV, Dordrecht, Netherlands). To imitate the pneumoperitoneum inside the abdomen, the pressure on the system was adjusted to $12 \mathrm{mmHg}$. A schematic image of the test setup is presented in Fig. 4.

The analog output of the sensor was digitized with an AD converter (Labjack U3). Another diaphragm pump from "ASF Thomas" with a flow of $1 \mathrm{l} / \mathrm{min}$ in combination with a custom-made filter house was used to determine whether particles entered the isolated volume. The particles were created with "toy smoke devices" (smoke balls) from a Chinese manufacturer. A flat cotton "make up" pad (Tippys, Paris, France) was used as a filter inside the custom-made filter house and a microscope was used to visually inspect the filter for particles after testing. 
For the dexterity tests, artificial skin material (Professional Skin Pad, Mk 2, Limbs \& Things, Bristol, United Kingdom) was clamped on the inside of a customized plastic model of the abdominal region.

\section{Software}

Besides the standard software needed to convert the signal from the ATM sensor to data in Matlab ${ }^{\circledR}$, a program was written for real-time visualization of the pressure inside the artificial pneumoperitoneum. During the smoke tests, two lines were visible on the screen. A red line indicated the desired pressure of the artificial pneumoperitoneum and a blue line the pressure inside the trocar. Other programs were written to store and plot data after the tests and to calibrate the ATM pressure sensor.

Test design—smoke tests

The goal of all tests is to prove that the Isolator System decreases the risk of infection. If so, the smoke tests must indicate that outside air does not enter the Isolator System. The smoke tests are performed inside a plastic container filled with thick smoke. This smoke consists of sticky

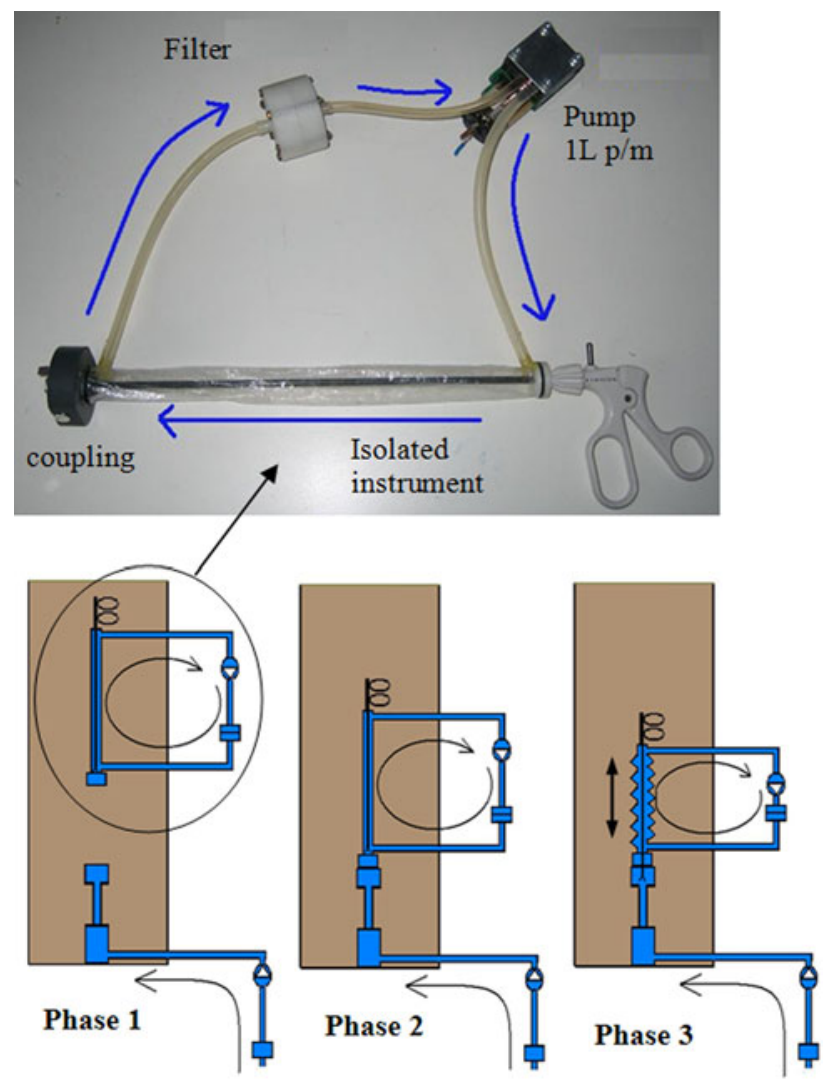

Fig. 5 Circulation of isolated volume through filter (top). Schematic view in chronological order of the test method (bottom) particles $\approx 1 \mu \mathrm{m}$ in diameter, which stick to everything they come into contact with. The isolated air circulates continuously through a filter during each test run (Fig. 5). As soon as smoke enters the Shaft Isolator due to failure of the Isolator System, the filter shows brown, sticky particles on its fibers. The complete smoke test consists of 9 runs of 5 min each. The 9 runs are divided into 8 test runs and 1 baseline run. During each test run, the isolated instrument is moved toward the trocar (Phase 1) and placed on the pressurized trocar (Phase 2). After the coupling is locked, the instrument is moved forward and backward in axial direction (Phase 3). This sequence is repeated five times during each test run. During the baseline test, the isolated instrument is removed and smoke from the container is directly filtered for $5 \mathrm{~min}$.

During the smoke tests, vision inside the container is blocked by thick smoke. To know whether the coupling connects properly onto the trocar, it is necessary to visualize the pressure in real time on a computer screen. After each test run, the filter is immediately removed from the filter house and stored in a sealed envelope. When the last test run is finished, the particles on four different defined areas of each filter are counted under microscopic magnification. Because the complete filter surface is known, the total number of particles on the filter can be roughly estimated by scaling the number of particles found on the defined areas.

\section{Test design—dexterity tests}

Dexterity tests were conducted on the plastic model of the abdominal region to investigate the intuitive design of the coupling. Therefore, three novices, students with knowledge about laparoscopic surgery, were asked to place the isolated trocar and to lock the isolated instrument on the

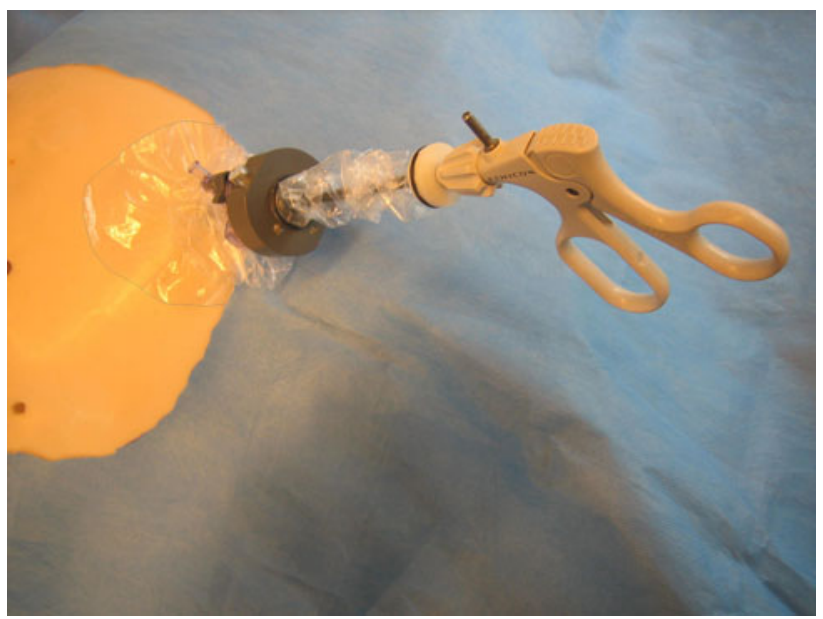

Fig. 6 Dexterity test setup: instrument handling was tested on a plastic model of the inflated abdominal area 
trocar. After the coupling was locked, the subjects were asked to move the instrument tip toward the model and rotate the instrument as far as possible around its pivot point. After approximately $10 \mathrm{~s}$, the subject was asked to unlock the system by using the single button on the coupling. They were not told that the button can only be pressed if the instrument was completely pulled out of the trocar and inside the Instrument Isolator. A schematic image of the test setup is presented in Fig. 6.

\section{Evaluation parameters}

The first parameter that was used for evaluation was the pressure inside the Isolator System during the test runs inside the smoke-filled container. The reason for this was to determine whether the coupling was properly connected and how the artificial pneumoperitoneum reacts on axial movements of the isolated instrument. The second and most important parameter was the number of particles that enter the isolated volume around the instrument shaft and tip. After each individual test run, the total amount of particles on a filter was roughly estimated after the particles inside four defined areas were counted. Because dimensions of the filter surface and total isolated volume are known, the particle density was easily calculated. Other parameters were the time needed to lock the isolated instrument onto the trocar and the time needed to unlock the instrument from the trocar. After the dexterity tests, the force needed to press the button of the coupling and the range of motion of the inserted instrument also were measured.

\section{Results}

\section{Isolator System development}

A number of design specifications were established for the Isolator System. In Table 1, column 3, the specifications of the actual design are presented in relation to the required value. Instrument insertion with one hand is possible. However, for fast and easy installation, two hands are required. Movements of the isolated trocar and instrument are similar to movements of an instrument and trocar in current practice. With the Isolator System, it is possible to insert a 330-mm-long instrument shaft for $300 \mathrm{~mm}$ into the trocar. Only one button is required to unlock the coupling for instrument changes. The isolated instrument locks automatically when placed correctly onto the trocar.

Evaluation—dexterity tests

Dexterity tests, conducted on the plastic model with novices as test subjects $(n=3)$, indicate that it takes an

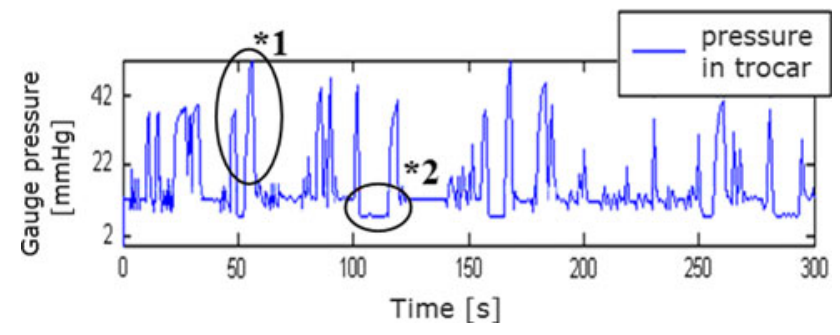

Fig. 7 Pressure inside trocar during tests. *1 Steep long spikes indicate that pressure vastly increases during closure of the shaft isolator valve, $* 2 \mathrm{U}$-shaped parts of the graph indicate that coupling is removed from the Isolated Trocar

Table 2 Estimated mean particles density inside isolated volume based on particles found on filters

\begin{tabular}{ll}
\hline Test number & Mean density $(\mathrm{p} / \mathrm{l})$ \\
\hline Test run 1, 3-7 & None found \\
Test run $2^{\mathrm{a}}$ & $0.3 \times 10^{5}$ \\
Baseline test & $1.0 \times 10^{10}$ \\
\hline
\end{tabular}

${ }^{a}$ Small leak due to loosening of shaft isolator

average of 3.1 ( $\mathrm{SD}, 0.74)$ seconds to lock the isolated instrument on the isolated trocar. The learning curve indicated that a novice requires roughly four attempts before the time to lock becomes constant. At the first attempt, two of the novices tried unsuccessfully to unlock the coupling from the trocar while the instrument was not completely pulled back inside the Shaft Isolator. Furthermore, it takes approximately $1 \mathrm{~min}$ of extra time to place the sticky pad of the Trocar Isolator correctly on the skin. All applied forces are within the design specification. Based on the tests performed with the design, it is estimated that the isolated instrument can be changed more than 200 times without failure.

Evaluation—smoke tests

During all test runs performed inside the smoke-filled container, the isolated instrument was successfully locked onto (and unlocked from) the isolated trocar 40 times. When the isolated instrument was locked on the isolated trocar, instrument movements in axial direction gave no complications. After the isolated instrument was replaced, the coupling locked itself in all cases. The coupling correctly unlocked as soon as the button was pressed and the instrument was completely retracted inside the shaft isolator. During all test runs, the coupling remained locked while the instrument was not completely inside the Shaft Isolator. Figure 7 presents the gauge pressure of the artificial pneumoperitoneum during one of seven successful test runs. 
In seven of eight test runs, no particles were found on the filters after magnification. During run 2, a leak occurred due to loosening of a part of the Shaft Isolator. During this test run, smoke entered the isolated instrument due to a poor fixation between the upper isolator flange and instrument shaft. This problem was fixed after the flange was properly replaced and the design was modified. Due to this small leak, this particular filter showed a small, light-brown spot with a diameter of $4 \mathrm{~mm}$. In the baseline test, the filter showed a 1mm-thick, dark-brown spot with a diameter of $8 \mathrm{~mm}$. Table 2 presents the roughly estimated particle density inside the isolated volume during each test run.

\section{Discussion}

A new Isolator System was developed for multiple laparoscopic and other minimally invasive procedures. This Isolator System protects exposed tissue against potentially harmful particles without restricting the surgeon performing the procedure. Not only can the patient benefit from the Isolator System, in case of contagious diseases, such as hepatitis or HIV, the Isolator System could protect the operating room and personnel against contaminating tissue and migrating cells coming from the patient [27]. In developing countries, this Isolator System could be used in operating rooms, intensive care units, or day care centers with limited resources. The question remains whether minimally invasive surgery in underdeveloped countries is realistic. Many studies indicate that, despite the under-resourced settings, it is possible and sometimes even cost efficient, to perform minimally invasive surgery in underdeveloped countries [28-31]. Already in India, in larger hospitals, day care centers, and camp settings, and wherever trained surgeons and gynecologists are available, the laparoscopic cholecystectomy and sterilization approach is frequently used because of its simplicity, safety, speed, and shorter postoperative hospital stay [29-32]. To investigate whether the Isolator System is useful for laparoscopic procedures in under-resourced settings, an additional study needs to be performed. This study also should indicate how many, and under which circumstances, procedures are performed in underdeveloped countries. Developed counties also can benefit from the new Isolator System. If the Isolator Systems is used in well-equipped hospitals or in combination with the use of a robotic surgical system, sealing of the cables, technical equipment, or robotic arms may no longer be necessary.

The dexterity and smoke tests that were performed with the prototype of the Isolator System show that it is possible to change isolated instruments safely during the procedure. Because no particles were found inside the isolated volume after testing, it is concluded that the new Isolator System can reduce the risk of contamination with harmful particles

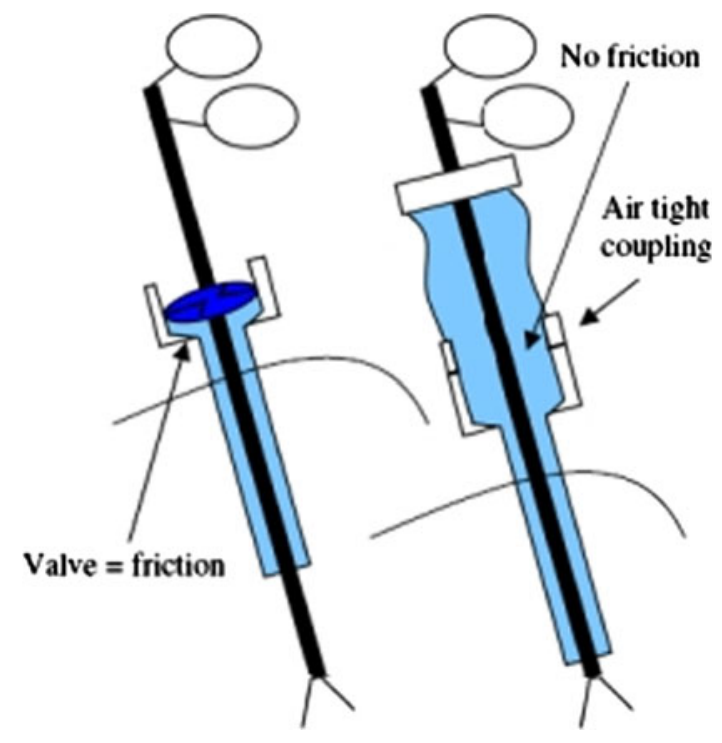

Fig. 8 Conventional trocar and instrument with friction between instrument shaft and trocar valve (left). Isolator System concept without friction between instrument shaft and trocar valve (right)

from outside. However, longer tests, similar to real procedures, must be performed by experienced surgeons. During the tests, professional equipment can quantify the sterility level of the system by counting the exact number of particles that enter the contained volume.

With the current design of the Isolator System, it is not yet possible to perform all types of laparoscopic surgery. If materials (e.g., needles, wires, clips) are used during the procedure or a large amount of tissue is retracted from the abdomen, adaptation of the design is necessary. Furthermore, the system can be modified such that the trocar valve automatically opens at the moment the coupling is locked on the trocar. If there is no contact between instrument shaft and trocar valve, friction is reduced to a minimum (Fig. 8). This greatly improves the haptic sensation of the surgeon.

Before placement of the first isolated trocar, a separate Veress needle is used to inflate the abdomen. In case the trocar pin of the Isolator System is equipped with a Veress mechanism or modified otherwise, controlled blind insertion should be possible. After the Trocar Isolator is removed at the end of a procedure, it can be necessary to close the incision with a small number of stitches or plaster pad. Further research is required to determine whether the Trocar Isolator can be equipped with means to close the incision from inside the Trocar Isolator.

\section{Conclusions}

The current Isolator System design for laparoscopy is affordable, easy to use, and easy to adapt for use in other 
MIS disciplines. This makes it interesting to use Isolator Systems in underdeveloped areas, intervention rooms, or outpatient clinics without the necessity of a sterile environment. Furthermore, in case of MIS performed on patients infected with a contagious disease, such as hepatitis or HIV, the Isolator System could act as an extra barrier that protects the operating room and personnel against contamination.

Acknowledgments The authors thank the BioMechanical Engineering (BME) technicians of the Delft University of Technology for help in designing and manufacturing the Isolator System prototype and test setup. They thank all surgeons and gynecologists for taking interest in this study and providing practical information about minimally invasive procedures.

Disclosures Tim Horeman, Frank-Willem Jansen, and Jenny Dankelman have no conflicts of interest or financial ties to disclose.

Open Access This article is distributed under the terms of the Creative Commons Attribution Noncommercial License which permits any noncommercial use, distribution, and reproduction in any medium, provided the original author(s) and source are credited.

\section{References}

1. Strickland AK, Martindale RG (2005) The increased incidence of intra-abdominal infections in laparoscopic procedures: potential causes, postoperative management, and prospective innovations. Surg Endosc 19:874-881

2. Moro ML, Carrieri MP, Tozzi AE, Lana S, Greco D (1996) Risk factors for surgical wound infections in clean surgery: a multicentre study. Ann Ital Chir 67:13-19

3. Zoutman D, McDonald S, Vethanayagan D (1998) Total and attributable costs of surgical wound infections at a Canadian tertiary-care centre. Infect Control Hosp Epidemiol 19:254-259

4. Kirkland KB, Briggs JP, Trivette SL, Wilkinson WE, Sexton DJ (1990) The impact of surgical-site infections in the 1990s: attributable mortality, excess length of hospitalization, and extra costs. Infect Control Hosp Epidemiol 20:725-730

5. Pagni S, Salloum EJ, Tobin GR, VanHimbergen DJ, Spence PA (1998) Serious wound infections after minimally invasive coronary bypass procedures. Ann Thorac Surg 66:92-94

6. Targarona EM, Balagué C, Knook MM, Trías M (2000) Trías laparoscopic surgery and surgical infection. Br J Surg 87:536544

7. den Hoed PT, Boelhouwer RU, Veen HF, Hopa WCJ, Bruining HA (1998) Infections and bacteriological data after laparoscopic and open gallbladder surgery. J Hosp Infect 39:27-37

8. Melling A, Ali B, Scott E, Leaper D (2001) Effects of preoperative warming on the incidence of wound infection after clean surgery: a randomized controlled trial. Lancet 358:876-880

9. Al-Abassi AA, Farghaly MM, Ahmed HL, Mobasher HLA, AlManee MS (2004) Infection after laparoscopic cholecystectomy: effects of infected bile and infected gallbladder wall. Br J Surg 167:268-273

10. Leaper DJ, Goor H, Reilly J, Petrosillo N, Geiss HK, Torres AJ, Berger A (2004) Surgical site infection: a European perspective of incidence and economic burden. Int Wound J 1:247-273

11. Brekalo Z, Innocenti P, Đuzel G, Liddo G, Ballone E, Šimunovi VJ (2007) Ten years of laparoscopic cholecystectomy: a comparison between a developed and a less developed country. Wein Klin Wochenschr 119:722-728

12. Joffe SN, Thomson WO, McGavigan J, Trexler PC (1978) A closed system surgical isolator for major elective abdominal operations. World J Surg 2:1432-2323

13. McLauchlan J, Pilcher MF, Trexler PC, Whalley RC (1974) The surgical isolator. Br Med J 1:323-324

14. Irwin W (1968) Apparatus for effecting surgical operations under improved aseptic conditions. Patent 1118657

15. Reyniers JA (1946) Surgical operating device. Patent 2403400

16. Scott FB, Fowler JM (1990) Isolator for use in surgery or as a clean room and method of using the same. Patent 4950222

17. Melles GRJ (2003) Operation unit. Patent 1462076

18. O'Connor, Cox ME (2004) Controlled environment device. Patent 20040116770

19. Tett KM (2005) Surgical tent and instrument set. Patent 2410440

20. Leahy PF et al (1979) Apparatus and method for use in surgery. Patent 5640977

21. Shenosky JT (2004) Collapsible, protective containment device. Patent 1488772

22. Kriek HR (2004) Sterile environments. Patent 2004/024014

23. Gravemaker R (2005) Controlling air quality around the operation field: design of a disposable surgical isolator. Final thesis, Delft University of Technology

24. Hoorman RWP (1985) Operation isolator. Final thesis, Delft University of Technology

25. Dankelman J, Grimbergen CA (2005) Systems approach to reduce errors in surgery. Surg Endosc 19:1017-1021

26. Cuschieri A (2006) Nature of human error: implications for surgical practice. Ann Surg 244:642-648

27. Champault G, Taffinder G, Ziol M, Riskalla H (1997) Cells are present in the smoke created during laparoscopic surgery. Br J Surg 84:993-995

28. Bal S, Reddy LGS, Parshad R, Guleria R, Kashyap L (2003) Feasibility and safety of day care laparoscopic cholecystectomy in a developing country. Postgrad Med J 79:284-288

29. Chauhan A, Mehrotra M, Bhatia PK, Baj B, Gupta AK (2006) Day care laparoscopic cholecystectomy: a feasibility study in a public health service hospital in a developing country. Department of Surgery, Base Hospital, Delhi Cantt, Delhi, 110010, India. World J Surg 30:1690-1695

30. Srimurthy KR, Ramesh AES (2006) Laparoscopic management of pediatric choledochal cysts in developing countries: review of ten cases. Pediatr Surg Int 22:144-149

31. Udwadia TE (2001) Navigating laparoscopic surgery into the next decade in developing countries-a personal perspective. Langenbecks Arch Surg 392:99-104

32. Mittal S (2003) Techniques for the interruption of tubal patency for female sterilization: RHL commentary. World Health Organization, The WHO Reproductive Health Library, Geneva 